\title{
OPTION REPLICATION IN DISCRETE TIME WITH THE COST OF ILLIQUIDITY*
}

\author{
YEGOR SOROKIN ${ }^{\dagger}$ AND HYEJIN KU ${ }^{\ddagger}$
}

\begin{abstract}
We introduce a model of liquidity risk through a stochastic supply curve for price taking traders. The supply curve gives the actual execution cost investors face in trading assets. We use the solutions to the modified Black-Scholes type PDE and obtain the delta-hedging strategies. We then show the replicating portfolio including liquidity costs converges to the payoff of the option. We demonstrate the replication error of discrete-time trading strategy decreases with inhomogeneous rebalancing times, and investigate an optimal positioning of them.
\end{abstract}

Key words. Option replication, inhomogeneous rebalancing, liquidity risk, illiquidity.

AMS subject classifications. 91B24, 91G20, 91G80.

\section{Introduction}

The pricing and hedging of contingent claims is a subject of paramount importance to the financial industry. Since the pioneering option pricing work of Black and Scholes, much research has been based on the simplified assumption of complete market. In a complete market it is possible to reproduce the payoff of any option using the replicating portfolio consisting of the underlying asset and cash. In reality, asset prices depend on a multitude of factors and there are restrictions on composition of portfolios the traders are allowed to hold. When market microstructure effects such as liquidity risk, microstructure noises, and information asymmetry are considered for more realistic models, it is not possible in general to replicate a contingent claim perfectly.

Liquidity risk is considered as the most important risk in finance industry these days. Traders' ability to buy or sell securities is limited or restricted, and they are not able to trade as much as they would prefer and cannot initiate or unwind positions instantly, especially in low-volume markets.

In the literature, the effect of illiquidity on the underlying asset is viewed in two ways. One is temporary price impact and the other is permanent price impact. The first approach focuses on the effect of liquidity cost on short time scale as the result of trading. The cost incurs while traders change their positions as a price-taker, and a trader cannot move the market by transactions. The second approach focuses on the effect of a large trader on the underlying asset (called feedback effect). By the large trader it means there is some lasting impact on the price evolution by its trading action. For example $[4,8,9,11,15]$ studied this effect.

This paper belongs to the first approach described above. Models for the effect of liquidity costs without considering permanent price impact have some similarities to those for non-linear transaction costs, in the sense that illiquidity adds some extra costs to trade. The effect of illiquidity makes trading more difficult or costly. Many researchers built on the model for market liquidity risk outlined in Cetin et al. [5]. Essentially the spot price of the underlying in the model depends on the size of the block being traded through the stochastic supply curve. For example if one wants to

*Received: December 24, 2014; accepted (in revised form): January 27, 2016. Communicated by David Cai.

${ }^{\dagger}$ Department of Mathematics and Statistics, York University, Market Risk Measurement, Scotiabank, Toronto, Canada. (yegor.sorokin@gmail.com)

$\ddagger$ Corresponding author: Department of Mathematics and Statistics, York University, Canada. (hku@mathstat.yorku.ca) 
purchase a huge amount of shares of stock, there may not be enough supply at the market price, so one will end up paying above the market price for such a big block of shares. In Cetin et al. [6], authors use strategies with minimal super-replication cost inclusive of liquidity premium to price contingent claims in continuous time setting. $\mathrm{Ku}$ et al. [12] derived a partial differential equation which provides discrete time delta hedging strategies whose expected hedging errors approach zero almost surely as the length of the revision interval goes to zero. The equation gives the value of the call from the seller's point of view. Rogers and Singh [16] also studied hedging of a European option with the effect of illiquidity costs. All these papers do not take into account the impact on the evolution of underlying asset from the actions of a trader.

For the literature on optimal liquidation in which the aim is to unwind an initial position by some fixed time horizon, we refer to [1,2], and [7]. These papers try to liquidate a given initial position "optimally" by some fixed time, and our problem is different from them since the aim is to replicate the random payoff at fixed time rather than liquidation. [14] considered the optimal portfolio choices in an illiquid market where the trading strategies were assumed to be of bounded variation. The paper of [3] discussed stock pinning on option expiration date and the price impact of delta-hedging.

This paper concerns minimizing the hedging error of a trading strategy under liquidity costs while replicating a European option. We use the solution of the partial differential equation of Black-Scholes type and define a class of discrete-time hedging strategies. We then extend the class of these trading strategies to include strategies with rehedgings located at varying distances from one another as in Grannan and Swindle [10]. We prove that the payoff of such a strategy converges in $L^{2}$ to the payoff of the option being replicated as the revision interval between rehedging times goes to zero. However, continuous hedging is not possible in practice and the number of available rebalancing times is limited. Thus selection of an optimal strategy that minimizes the replication error under liquidity costs is an important question.

The main contribution of this paper is to compute moreover the exact first order term of the mean squared hedging error (MSHE) with varying rebalancing times, according to the interval size $\Delta t$ of equally-spaced times

$$
\operatorname{MSHE}=A\left(d(\cdot) ; d^{\prime}(\cdot)\right) \Delta t+O\left(\Delta t^{2}\right),
$$

where $d$ is a suitable function that maps the equidistant times $(i \Delta t)_{i}$ to the varying rehedging times $\left(t_{i}\right)_{i}: d\left(t_{i}\right)=i \Delta t$. Then the term $A\left(d(\cdot) ; d^{\prime}(\cdot)\right)$ is minimized (over all deterministic functions $d(\cdot))$ using the calculus of variations, thus giving an optimal positioning of the rebalancing times.

The article is organized as follows. Section 2 explains the model and presents the main results of the paper. Section 3 studies a selection of optimal strategy which minimizes the mean squared replication error including liquidity costs. Section 4 presents some conclusions. The proofs of theorems are provided in Appendix A.

\section{Main results}

Consider a filtered probability space $\left(\Omega, \mathcal{F},\left(\mathcal{F}_{t}\right)_{0 \leq t \leq T}, \mathbb{P}\right)$ satisfying the usual conditions, where $T$ is a fixed time, and $\mathbb{P}$ represents the statistical or empirical probability measure. We assume a market with a stock and a money market account. We assume the stock pays no dividends, and the rate of interest is zero.

We consider a stochastic supply curve $S(t, x, \omega)$ which represents the stock price per share at time $t \in[0, T]$ that a trader pays/receives for an order of size $x \in \mathbb{R}$ as a price taker. A positive order $(x>0)$ represents a buy, a negative order $(x<0)$ signifies a sale, and $x=0$ corresponds to the marginal trade. $S(t, x, \omega)$ is assumed measurable with 
respect to the filtration $\left(\mathcal{F}_{t}\right)$, nonnegative, and non-decreasing in $x$ almost surely. For the detailed structure of the supply curve, we refer to Cetin et al. [5] where the authors define the self-financing condition in a general setting and connect to the arbitrage pricing theory.

Let $S(t, 0, \omega)=S_{t}$ be the marginal price of the supply curve. We assume the price process $S_{t}$ follows a geometric Brownian motion

$$
d S_{t}=\mu S_{t} d t+\sigma S_{t} d W_{t}, \quad 0 \leq t \leq T,
$$

where the drift $\mu$ is a constant, the volatility $\sigma$ is a positive real number, $W$ is a standard Brownian motion, and $T$ is the terminal time of a European contingent claim with the payoff $p\left(S_{T}\right)$. We also assume a multiplicative supply curve

$$
S(t, x, \omega)=f(x) S_{t},
$$

where $f$ is a smooth and increasing function with $f(0)=1$. Then the slope at 0 of supply curve, $f^{\prime}(0)$ (denoted by $\alpha$ ) is interpreted as the parameter for liquidity risk. The parameter $\alpha$ is estimated using simple regression methodology from the history of stock prices. ${ }^{1}$ A trading strategy is a pair $\left(X_{t}, Y_{t}: t \in[0, T]\right)$ where $X_{t}$ represents the number of units of stock held at time $t$ and $Y_{t}$ represents the trader's cash holding at time $t$. $X_{t}$ and $Y_{t}$ are predictable and optional processes respectively, to the filtration $\left(\mathcal{F}_{t}\right)_{0 \leq t \leq T}$.

Let us consider equally spaced times $0=t_{0} \leq t_{1} \leq t_{2} \leq \cdots \leq t_{n}=T$. Set $\Delta t=t_{i}-t_{i-1}$ for $i=1, \ldots, n$. The following definition describes a self-financing condition for discrete strategies with liquidity risk.

Definition 2.1. A discrete self-financing trading strategy is a trading strategy $\left(X_{t}, Y_{t}\right.$ : $\left.t \in\left\{t_{i}\right\}_{i=0}^{n}\right)$ which satisfies

$$
Y_{t}=Y_{0}+X_{0} S\left(0, X_{0}\right)+\sum_{t_{i}<t} X_{t_{i}}\left(S_{t_{i+1}}-S_{t_{i}}\right)-\sum_{t_{i}<t} \Delta X_{t_{i}}\left[S\left(t_{i}, \Delta X_{t_{i}}\right)-S\left(t_{i}, 0\right)\right],
$$

where $\Delta X_{t_{i}}=X_{t_{i}}-X_{t_{i-1}}(i \geq 1)$ and $\Delta X_{t_{0}}=X_{0}$.

Considering the usual self-financing condition without liquidity costs, we define the accumulated liquidity costs of a discrete trading strategy $\left(X_{t}, Y_{t}: t \in\left\{t_{i}\right\}_{i=0}^{n}\right)$ by

$$
L_{t}=\sum_{t_{i}<t} \Delta X_{t_{i}}\left[S\left(t_{i}, \Delta X_{t_{i}}\right)-S\left(t_{i}, 0\right)\right]
$$

and the total liquidity costs up to time $T$ is

$$
L_{T}=\sum_{i=0}^{n-1} \Delta X_{t_{i}}\left[S\left(t_{i}, \Delta X_{t_{i}}\right)-S\left(t_{i}, 0\right)\right]
$$

where $\Delta X_{t_{i}}=X_{t_{i}}-X_{t_{i-1}}$ for $i \geq 1$ and $\Delta X_{t_{0}}=X_{0}$.

In practice, continuous hedging is not possible, thus one cannot replicate the option perfectly and must accept an error. Letting $C_{0}$ denote the value at time 0 of contingent claim being replicated, the hedging error inclusive of liquidity costs is

$$
H E=C_{0}+\sum_{i=0}^{n-1} X_{t_{i}}\left(S_{t_{i+1}}-S_{t_{i}}\right)-L_{T}-p\left(S_{T}\right) .
$$

\footnotetext{
${ }^{1}$ The value of $\alpha$ turns out to be small in most cases, usually with $0<\alpha<0.001$.
} 
Hedging errors are random, so we consider distributions of hedging errors. We compare efficiency of the different replicating trading strategies by looking at the corresponding distributions of the hedging errors and their parameters. We use the mean squared hedging error (=the variance of hedging error) for a criterion to estimate the hedging error.

DEFINITION 2.2. The mean squared hedging error (MSHE) of a replicating trading strategy is

$$
\mathbb{E}\left[C_{0}+\sum_{i=0}^{n-1} X_{t_{i}}\left(S_{t_{i+1}}-S_{t_{i}}\right)-L_{T}-p\left(S_{T}\right)\right]^{2}
$$

Not only did Black and Scholes show how to compute the fair price of the European option, but also they laid out the method for eliminating the risk of writing an option through continuous delta hedging. Implementation of continuous delta-hedging amounts to maintaining and rebalancing two positions with a money market account (in cash) and a stock. The goal of delta-hedging is to eliminate the risk of writing an option completely (continuous hedging in theory) or at least significantly reduce the level of risk (discrete hedging in practice).

Leland [13] investigated the hedging error over each revision interval with the presence of transaction costs, and modified the parameter of Black-Scholes price for deltahedging strategies. Ku et al. [12] argue that a dynamic delta hedging according to their partial differential equation for a European contingent claim produces hedging errors over the period $[0, T]$ whose expectation approaches 0 as the length of the revision interval goes to 0 . They also show that the payoff of the discrete replicating trading strategy converges almost surely to the terminal payoff of the option $p\left(S_{T}\right)$. In these papers the trading times are equally spaced over the life of the option.

Considering alternatives to equally spaced trading times over the life of an option, the hedging error can be improved. We proceed to work with trading strategies in homogenous rehedging times. We parametrize varying rebalancing times with a smooth, positive, strictly increasing function $d(t)$ via $t_{i}=d^{-1}(i \Delta t)$. We also require that $d(0)=$ $0, d(T)=T$ and $d^{\prime}(t) \neq 0$. In other words one may recover the positioning of rehedging times through inverse of $d(t)$. Taking $d(t)=t$ corresponds to the constant interval case, equally spaced trading dates. Different functions $d(t)$ yield different locations of the rehedging times $t_{i}$. Figure 2.1 shows an example of $d(t)$, where the left half of the graph is concave down (which translates into more frequent rehedging at the beginning) and the right half of the graph is convex (which corresponds to more frequent rehedging toward the end of a period).

THEOREM 2.1. Consider the discrete time delta-hedging strategy $X_{t_{i}}=C_{S}\left(t_{i}, S_{t_{i}}\right)$ where $C$ is the solution of the $P D E^{2}$

$$
C_{t}(t, S)+1 / 2 \sigma^{2} S^{2} C_{S S}(t, S)\left[1+2 \alpha S C_{S S}(t, S)\right]=0
$$

\footnotetext{
${ }^{2}$ The existence and uniqueness of solutions for convex payoffs can be shown in the viscosity sense, by comparing to Equation (1.1) in Cetin et al. [6] with $l=\frac{1}{4 \alpha S}$. However, the existence of a classical solution is an open issue. The main problem is that the term derived from the effect of illiquidity, $\alpha \sigma^{2} S^{3}\left(C_{S S}(t, S)\right)^{2}$, in this fully nonlinear PDE is hard to deal analytically; it is very difficult to solve the well-posedness issue in the classical sense. The numerical solutions to this PDE under the smoothness hypotheses are computed in Section 3, and the numerical results we obtained are consistent with the theory, suggesting that this assumption does not cause any harm.
} 


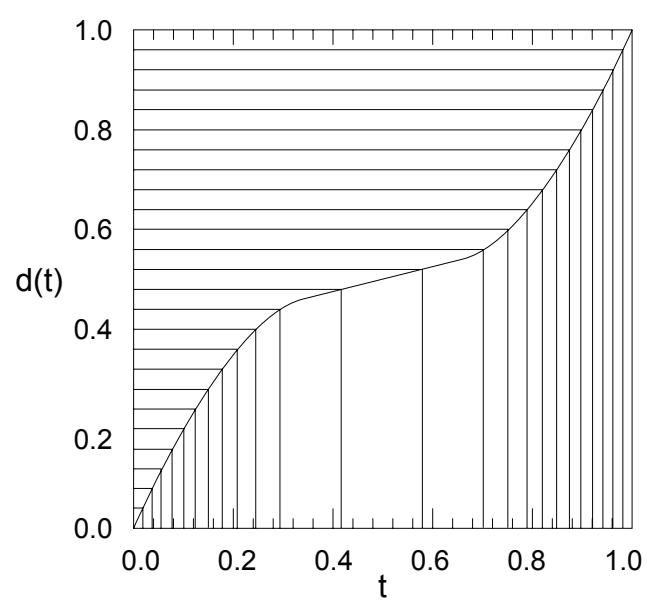

FIG. 2.1. Setting up the varying rehedging times. Steeper slope of $d(t)$ corresponds to more frequent rebalancing.

with the final condition $C(T, S)=p\left(S_{T}\right)$, we have

$$
C\left(0, S_{0}\right)+\sum_{t_{i}<T} C_{S}\left(t_{i}, S_{t_{i}}\right)\left(S_{t_{i+1}}-S_{t_{i}}\right)-\sum_{t_{i}<T} \Delta X_{t_{i}}\left[S\left(t_{i}, \Delta X_{t_{i}}\right)-S\left(t_{i}, 0\right)\right] \rightarrow p\left(S_{T}\right)
$$

in $L^{2}$-sense as $\Delta t \rightarrow 0$. In other words, the MSHE of this discrete delta-hedging strategy approaches zero.

Proof. See Appendix A.

Lemma 2.1. Let $C(t, S)$ be a solution to

$$
C_{t}(t, S)+1 / 2 \sigma^{2} S^{2} C_{S S}(t, S)\left[1+2 \alpha S C_{S S}(t, S)\right]=0
$$

with the final condition $C(T, S)=p\left(S_{T}\right)$, then ${ }^{3}$

$$
\begin{aligned}
\sum_{t_{i}<T} C_{S}\left(t_{i}, S_{t_{i}}\right)\left(S_{t_{i+1}}-S_{t_{i}}\right) & \rightarrow \int_{0}^{T} C_{S}\left(t, S_{t}\right) d S_{t} \\
L_{T} & \rightarrow \int_{0}^{T} \sigma^{2} f^{\prime}(0) S_{t}^{3} C_{S S}^{2}\left(t, S_{t}\right) d t
\end{aligned}
$$

in $L^{2}$-sense as $\Delta t \rightarrow 0$.

Proof. See Appendix A.

The next theorem gives the coefficient of $\Delta t$ as a leading order term of the mean squared hedging error assuming varying rehedging times.

ThEOREM 2.2. Consider the discrete time delta-hedging strategy $X_{t_{i}}=C_{S}\left(t_{i}, S_{t_{i}}\right)$ where $C$ is the solution of the PDE in Lemma 2.1,

$$
\mathbb{E}\left[C\left(0, S_{0}\right)+\sum_{t_{i}<T} C_{S}\left(t_{i}, S_{t_{i}}\right)\left(S_{t_{i+1}}-S_{t_{i}}\right)-\sum_{t_{i}<T} \Delta X_{t_{i}}\left(S\left(t_{i}, \Delta X_{t_{i}}\right)-S\left(t_{i}, 0\right)\right)-p\left(S_{T}\right)\right]^{2}
$$

\footnotetext{
${ }^{3}$ We assume smoothness conditions on $C(t, x)$, i.e., $\|C\|_{m, n, p}=\sup \left[x^{m} \frac{\partial^{p+n} C(t, x)}{\partial t^{p} \partial x^{n}}\right]$ is finite for all
} nonnegative $m, n$, and $p$. 


$$
=\Delta t \mathbb{E}\left[\int_{0}^{T} \sigma^{4} \frac{1}{d^{\prime}(t)} C_{S S}^{2}\left(t, S_{t}\right) S_{t}^{4}\left(\frac{1}{2}+2 \alpha C_{S S}\left(t, S_{t}\right) S_{t}+2 \alpha^{2} C_{S S}^{2}\left(t, S_{t}\right) S_{t}^{2}\right) d t\right]+O\left(\Delta t^{2}\right)
$$

Proof. See Appendix A.

REMARK 2.1. In comparison with the settings in Cetin et al. [6], the class of deltahedging strategies suggested in our paper satisfies the condition for admissible trading strategies in Cetin et al. [6], since the number of shares held at each time $t$ in the portfolio is $C_{S}(S, t)$ and it can be written in the form of Equation (2.2) in Cetin et al. [6].

\section{Selection of optimal strategies}

In this section we show that the hedging errors are reduced by using varying instead of equally spaced rehedging times. We wish to replicate the payoff $p\left(S_{T}\right)$ of a European option with a fixed initial capital prescribed by the partial differential equation and a fixed number of available rebalancing times. Since continuous hedging is not possible, there must be a tracking error. We are minimizing the mean squared replication error, that is the first order term in $\Delta t$ from Theorem 2.2. Thus we investigate the optimal positioning of the rehedging times over all deterministic functions $d(t)$ :

$$
\min _{d(t)} \mathbb{E}\left[\int_{0}^{T} \sigma^{4} \frac{1}{d^{\prime}(t)} C_{S S}^{2}\left(t, S_{t}\right) S_{t}^{4}\left(\frac{1}{2}+2 \alpha C_{S S}\left(t, S_{t}\right) S_{t}+2 \alpha^{2} C_{S S}^{2}\left(t, S_{t}\right) S_{t}^{2}\right) d t\right] .
$$

We employ the calculus of variations to solve the minimization problem. Essentially we find a solution $d(t)$ giving the location of the rehedging times that will minimize the hedging error. We start by transforming the coefficient in front of $\Delta t$ in the mean squared hedging error using Fubini's theorem

$$
\begin{aligned}
& \mathbb{E}\left[\int_{0}^{T} \sigma^{4} \frac{1}{d^{\prime}(t)} C_{S S}^{2}\left(t, S_{t}\right) S_{t}^{4}\left(\frac{1}{2}+2 \alpha C_{S S}\left(t, S_{t}\right) S_{t}+2 \alpha^{2} C_{S S}^{2}\left(t, S_{t}\right) S_{t}^{2}\right) d t\right] \\
= & \sigma^{4} \int_{0}^{T} \frac{1}{d^{\prime}(t)}\left(\frac{1}{2} \mathbb{E}\left[C_{S S}^{2}\left(t, S_{t}\right) S_{t}^{4}\right]+2 \alpha \mathbb{E}\left[C_{S S}^{3}\left(t, S_{t}\right) S_{t}^{5}\right] d t+2 \alpha^{2} \mathbb{E}\left[C_{S S}^{4}\left(t, S_{t}\right) S_{t}^{6}\right]\right) d t .
\end{aligned}
$$

Denote by $A(t)$ the expression in parentheses, that is,

$$
A(t)=\frac{1}{2} \mathbb{E}\left[C_{S S}^{2} S_{t}^{4}\right]+2 \alpha \mathbb{E}\left[C_{S S}^{3} S_{t}^{5}\right]+2 \alpha^{2} \mathbb{E}\left[C_{S S}^{4} S_{t}^{6}\right]
$$

Recall that in the calculus of variations the problem of finding a function $d(t)$ which minimizes the following integral

$$
I(d)=\int_{t_{1}}^{t_{2}} F\left(t, d(t), d^{\prime}(t)\right) d t
$$

where $d\left(t_{1}\right)=d_{1}, d\left(t_{2}\right)=d_{2}$, and $d^{\prime}(t) \neq 0$. We want to solve

$$
\int_{0}^{T} \frac{1}{d^{\prime}(t)} A(t) d t
$$

with $d(0)=0, d(T)=T, d^{\prime}(t) \neq 0$, which means that the integrand

$$
F\left(t, d(t), d^{\prime}(t)\right)=\frac{1}{d^{\prime}(t)} A(t)
$$


in our case. The functional $F$ depends only on $t$ and $d^{\prime}$, and the Euler's equation is $\frac{d}{d t} F_{d^{\prime}}\left(t, d^{\prime}\right)=0$. Next we integrate the Euler's equation with respect to $t$ from both sides to get rid of the derivative in $t$. Then $F_{d^{\prime}}\left(t, d^{\prime}\right)=C_{1}$ for some constant $C_{1}$.

Differentiating $F$ with respect to $d^{\prime}$,

$$
F_{d^{\prime}}\left(t, d^{\prime}\right)=-\frac{1}{\left(d^{\prime}(t)\right)^{2}} A(t)=C_{1}
$$

Then we have $0 \leq\left(d^{\prime}(t)\right)^{2}=-\frac{A(t)}{C_{1}}$. Next we take the square root on both sides for $d^{\prime}(t)$ :

$$
d^{\prime}(t)= \pm \sqrt{\frac{|A(t)|}{\left|-C_{1}\right|}}= \pm \frac{\sqrt{|A(t)|}}{\sqrt{\left|-C_{1}\right|}} .
$$

In order to find a value of $\sqrt{\left|-C_{1}\right|}$ from the boundary condition, we integrate with respect to $t$ from both sides. Then

$$
d(t)= \pm \int_{0}^{t} \sqrt{\frac{|A(x)|}{\left|-C_{1}\right|}} d x+C_{2}
$$

Recall that we require $d(t)$ to be positive, so one is interested only in $d(t)=$ $+\int_{0}^{t} \sqrt{\frac{|A(x)|}{\left|-C_{1}\right|}} d x+C_{2}$. Now we use the boundary conditions to determine the values for $C_{1}$ and $C_{2}$. First we make use of $d(0)=0$.

$$
d(0)=0=\int_{0}^{0} \sqrt{\frac{|A(x)|}{\left|-C_{1}\right|}} d x+C_{2}=0+C_{2}
$$

so $C_{2}=0$ and $d(t)=+\int_{0}^{t} \sqrt{\frac{|A(x)|}{\left|-C_{1}\right|}} d x$. Now using $d(T)=T$,

$$
d(T)=T=\int_{0}^{T} \sqrt{\frac{|A(x)|}{\left|-C_{1}\right|}} d x=\frac{1}{\sqrt{\left|-C_{1}\right|}} \int_{0}^{T} \sqrt{|A(x)|} d x
$$

thus

$$
\frac{1}{\sqrt{\left|-C_{1}\right|}}=\frac{T}{\int_{0}^{T} \sqrt{|A(x)|} d x} .
$$

The final form of $d(t)$ is

$$
\begin{aligned}
d(t) & =\int_{0}^{t} \sqrt{\frac{|A(x)|}{\left|-C_{1}\right|}} d x \\
& =\frac{T}{\int_{0}^{T} \sqrt{|A(x)|} d x} \int_{0}^{t} \sqrt{|A(x)|} d x .
\end{aligned}
$$

Now we discuss the numerical solution to the problem and present some simulations. We consider the hedging problem of a standard European call and compute $d(t)$ numerically for the call option. Not all the smoothness hypotheses that we imposed to prove our results is fulfilled for the call since the derivatives have singularities at point $(T, K)$, but this does not imply that our results do not cover this case. The formula 
for the first order term to reduce MSHE, derived in Theorem 2.2, is used to approximate hedging error of call options. The numerical results we obtain in this section are consistent with the theory.

Table 3.1 shows the improvement in hedging errors from using varying rehedging times instead of equally spaced ones. The computations are made for the European call option with 250 rehedging times (that is, daily rebalancing), maturity $T=1$ (a year), $\sigma=0.1$, and the spot price of the underlying $S_{0}=100$. We note that the hedging errors in the Black-Scholes setting (the case when $\alpha=0$ ) is also reduced by using varying rehedging times as well.

\begin{tabular}{clccccc}
\hline \multirow{2}{*}{$\alpha$} & \multirow{2}{*}{ strike } & \multirow{2}{*}{ call price } & \multicolumn{4}{c}{ mean } \\
\cline { 4 - 7 } & & & $\mathrm{HE}_{e}$ & $\mathrm{HE}_{v}$ & $\mathrm{MSHE}_{e}$ & $\mathrm{MSHE}_{v}$ \\
\hline 0.0001 & 95 & 6.88143273 & 0.000227 & 0.000132 & 0.005185 & 0.004467 \\
& 100 & 3.97951947 & 0.000162 & 0.000096 & 0.009780 & 0.008972 \\
& 105 & 2.05702452 & 0.000214 & 0.000198 & 0.001727 & 0.001041 \\
\hline 0.0005 & 95 & 6.78019826 & 0.000247 & 0.000342 & 0.009105 & 0.007433 \\
& 100 & 3.95192241 & 0.000519 & 0.000381 & 0.009368 & 0.006045 \\
& 105 & 2.04212433 & 0.000229 & 0.000201 & 0.003033 & 0.001660 \\
\hline 0.001 & 95 & 6.86598135 & 0.000114 & 0.000197 & 0.005159 & 0.004590 \\
& 100 & 3.96012972 & 0.000336 & 0.000224 & 0.008562 & 0.005249 \\
& 105 & 2.03966783 & 0.000032 & 0.000439 & 0.003024 & 0.002197 \\
\hline
\end{tabular}

TABLE 3.1. Comparison of the hedging errors for equally spaced and varying rehedging times (subscripts e and $v$ correspondingly).

Figures 3.1 and 3.2 present the optimal $d(t)$. The parameter values used in this computation are initial spot price $S_{0}=100, T=0.5$ (expiration in half a year), $\sigma=0.4$, and $\alpha=0.0001$. We observe the graph is convex. We plot $d(t)$ in Figure 3.1 for strikes 100, 90, and 80, while Figure 3.2 displays $d(t)$ for strikes 100, 110, and 120. As the option gets deeper in the money or out of the money, the recommendation is to rebalance more frequently toward the expiration (that is, the function's shape becomes steeper).
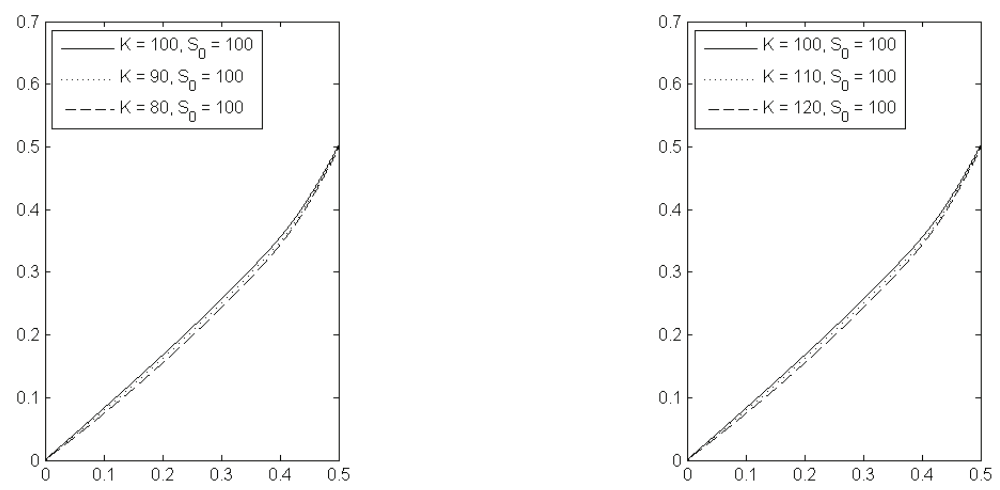

FIG. 3.1. Optimal d(t) for a Euro call for decreasing strikes.
FIG. 3.2. Optimal $d(t)$ for a Euro call for increasing strikes. 


\section{Conclusions}

We investigated the discrete time hedging problem of a contingent claim under liquidity risk. We modeled liquidity costs via a stochastic supply curve with an underlying asset price depending on order flow, that is purchases are executed at higher prices while sales are executed at lower prices. We used a partial differential equation to define a delta-hedging strategy and showed that the payoff of this discrete replicating strategy converges to the payoff of the option as the length of revision interval goes to zero. Since continuous hedging is not possible in practice, we introduced the class of discrete deltahedging trading strategies with varying rebalancing times and showed the mean squared hedging error is improved with varying instead of equally spaced rehedging times. It is advised to rebalance more frequently toward the expiration date as the option gets deeper in the money or out of the money.

\section{Appendix A. Proofs of the theorems.}

LEMMA A.1.

$$
\sum_{t_{i}<T} C_{S}\left(t_{i}, S_{t_{i}}\right)\left(S_{t_{i+1}}-S_{t_{i}}\right) \longrightarrow \int_{0}^{T} C_{S}\left(t, S_{t}\right) d S_{t}
$$

in $L^{2}$-sense as $\Delta t \rightarrow 0$.

The result of Lemma A.1 is well-known and can be proved in more general setting, but we present the proof in here since we need the explicit expression of the leading order term of errors for the proof of Theorem 2.2 .

Proof. Consider

$$
\begin{aligned}
& \mathbb{E}\left[\sum_{t_{i}<T} C_{S}\left(t_{i}, S_{t_{i}}\right)\left(S_{t_{i+1}}-S_{t_{i}}\right)-\int_{0}^{T} C_{S}\left(t, S_{t}\right) d S_{t}\right]^{2} \\
= & \mathbb{E}\left[\sum_{t_{i}<T} \int_{t_{i}}^{t_{i+1}} C_{S}\left(t_{i}, S_{t_{i}}\right) d S_{t}-\sum_{t_{i}<T} \int_{t_{i}}^{t_{i+1}} C_{S}\left(t, S_{t}\right) d S_{t}\right]^{2} \\
= & \mathbb{E}\left[\sum_{t_{i}<T} \int_{t_{i}}^{t_{i+1}}\left(C_{S}\left(t_{i}, S_{t_{i}}\right)-C_{S}\left(t, S_{t}\right)\right) d S_{t}\right]^{2} \\
= & \mathbb{E}\left[\sum_{i=j}\left(\int_{t_{i}}^{t_{i+1}}\left(C_{S}\left(t_{i}, S_{t_{i}}\right)-C_{S}\left(t, S_{t}\right)\right) d S_{t}\right)^{2}\right] \\
& \quad+\mathbb{E}\left[\sum_{i \neq j} \int_{t_{i}}^{t_{i+1}}\left(C_{S}\left(t_{i}, S_{t_{i}}\right)-C_{S}\left(t, S_{t}\right)\right) d S_{t} \int_{t_{j}}^{t_{j+1}}\left(C_{S}\left(t_{j}, S_{t_{j}}\right)-C_{S}\left(t, S_{t}\right)\right) d S_{t}\right] .
\end{aligned}
$$

The cross terms (the second term of the last equation) become zero. We work with the diagonal terms first.

$$
\begin{aligned}
& \mathbb{E}\left[\sum_{i}\left(\int_{t_{i}}^{t_{i+1}}\left(C_{S}\left(t_{i}, S_{t_{i}}\right)-C_{S}\left(t, S_{t}\right)\right) d S_{t}\right)^{2}\right] \\
= & \mathbb{E}\left[\sum_{i}\left(\int_{t_{i}}^{t_{i+1}}\left[C_{S}\left(t_{i}, S_{t_{i}}\right)-C_{S}\left(t, S_{t}\right)\right]\left[\mu S_{t} d t+\sigma S_{t} d W_{t}\right]\right)^{2}\right]
\end{aligned}
$$


1956 OPTION REPLICATION IN DISCRETE TIME WITH THE COST OF ILLIQUIDITY

$$
=\mathbb{E}\left[\sum_{i}\left(\int_{t_{i}}^{t_{i+1}}\left[C_{S}\left(t_{i}, S_{t_{i}}\right)-C_{S}\left(t, S_{t}\right)\right] \mu S_{t} d t+\int_{t_{i}}^{t_{i+1}}\left[C_{S}\left(t_{i}, S_{t_{i}}\right)-C_{S}\left(t, S_{t}\right)\right] \sigma S_{t} d W_{t}\right)^{2}\right]
$$

Now we demonstrate that the order of each sum is greater than or equal to $O(\Delta t)$ to conclude that the whole expression converges to zero. We start by showing that the sum of squares of the second term has expectation $O(\Delta t)$, that is

$$
\mathbb{E}\left[\sum_{i}\left(\int_{t_{i}}^{t_{i+1}}\left[C_{S}\left(t_{i}, S_{t_{i}}\right)-C_{S}\left(t, S_{t}\right)\right] \sigma S_{t} d W_{t}\right)^{2}\right]=O(\Delta t) \rightarrow 0 \quad(\text { as } \Delta t \rightarrow 0) .
$$

To prove this we consider

$$
\begin{aligned}
& \mathbb{E}\left[\sum_{i}\left(\int_{t_{i}}^{t_{i+1}}\left[C_{S}\left(t_{i}, S_{t_{i}}\right)-C_{S}\left(t, S_{t}\right)\right] \sigma S_{t} d W_{t}\right)^{2}\right] \\
= & \mathbb{E}\left[\sum_{i} \mathbb{E}_{\mathcal{F}_{t_{i}}}\left[\int_{t_{i}}^{t_{i+1}}\left[C_{S}\left(t_{i}, S_{t_{i}}\right)-C_{S}\left(t, S_{t}\right)\right] \sigma S_{t} d W_{t}\right]^{2}\right] \\
= & \mathbb{E}\left[\sum_{i} \mathbb{E}_{\mathcal{F}_{t_{i}}}\left[\int_{t_{i}}^{t_{i+1}}\left(C_{S}\left(t_{i}, S_{t_{i}}\right)-C_{S}\left(t, S_{t}\right)\right)^{2} \sigma^{2} S_{t}^{2} d t\right]\right]
\end{aligned}
$$

by the Itô isometry (see for instance [17]). Using a Taylor series expansion,

$$
\begin{aligned}
& \mathbb{E}\left[\sum_{i} \mathbb{E}_{\mathcal{F}_{t_{i}}}\left[\int_{t_{i}}^{t_{i+1}}\left(C_{S S}^{2}\left(t_{i}, S_{t_{i}}\right)\left(S_{t}-S_{t_{i}}\right)^{2}+O\left(\Delta t^{3 / 2}\right)\right) \sigma^{2} S_{t}^{2} d t\right]\right] \\
= & \mathbb{E}\left[\sum_{i}\left(\mathbb{E}_{\mathcal{F}_{t_{i}}}\left[\int_{t_{i}}^{t_{i+1}} C_{S S}^{2}\left(t_{i}, S_{t_{i}}\right)\left(S_{t}-S_{t_{i}}\right)^{2} \sigma^{2} S_{t}^{2} d t\right]+O\left(\Delta t^{5 / 2}\right)\right)\right] \\
= & \mathbb{E}\left[\sum_{i}\left(\sigma^{2} C_{S S}^{2}\left(t_{i}, S_{t_{i}}\right) \int_{t_{i}}^{t_{i+1}} \mathbb{E}_{\mathcal{F}_{t_{i}}}\left[\left(S_{t}-S_{t_{i}}\right)^{2} S_{t}^{2}\right] d t+O\left(\Delta t^{5 / 2}\right)\right)\right] .
\end{aligned}
$$

We note that the fact

$$
\mathbb{E}_{\mathcal{F}_{t_{i}}}\left[\left(S_{t}-S_{t_{i}}\right)^{2} S_{t}^{2}\right]=\sigma^{2} S_{t_{i}}^{4}\left(t-t_{i}\right)+O\left(t-t_{i}\right)^{2}
$$

which can be directly calculated from the distribution of $S_{t}$. Then Equation (A.2) equals

$$
\begin{aligned}
& \mathbb{E}\left[\sum_{i}\left(\sigma^{2} C_{S S}^{2}\left(t_{i}, S_{t_{i}}\right) \int_{t_{i}}^{t_{i+1}} \mathbb{E}_{\mathcal{F}_{t_{i}}}\left[\left(S_{t}-S_{t_{i}}\right)^{2} S_{t}^{2}\right] d t+O\left(\Delta t^{5 / 2}\right)\right)\right] \\
= & \mathbb{E}\left[\sum_{i}\left(\sigma^{2} C_{S S}^{2}\left(t_{i}, S_{t_{i}}\right)\left[\sigma^{2} S_{t_{i}}^{4} \int_{t_{i}}^{t_{i+1}}\left(t-t_{i}\right) d t+O\left(\Delta t^{3}\right)\right]+O\left(\Delta t^{5 / 2}\right)\right)\right] \\
= & \mathbb{E}\left[\sum_{i}\left(\frac{\sigma^{4}}{2} C_{S S}^{2}\left(t_{i}, S_{t_{i}}\right) S_{t_{i}}^{4}\left(t_{i+1}-t_{i}\right)^{2}+O\left(\Delta t^{5 / 2}\right)\right)\right] \\
= & \mathbb{E}\left[\sum_{i}\left(O\left(\Delta t^{2}\right)+O\left(\Delta t^{5 / 2}\right)\right)\right]=O(\Delta t) \rightarrow 0 \quad(\text { as } \Delta t \rightarrow 0) .
\end{aligned}
$$


This is the only term with order exactly $O(\Delta t)$. To characterize the convergence we find that

$$
\begin{aligned}
& \mathbb{E}\left[\sum_{i} \frac{\sigma^{4}}{2} C_{S S}^{2}\left(t_{i}, S_{t_{i}}\right) S_{t_{i}}^{4}\left(t_{i+1}-t_{i}\right)^{2}\right] \\
= & \mathbb{E}\left[\sum_{i} \frac{\sigma^{4}}{2} C_{S S}^{2}\left(t_{i}, S_{t_{i}}\right) S_{t_{i}}^{4}\left(t_{i+1}-t_{i}\right)\left(t_{i+1}-t_{i}\right)\right] \\
= & \mathbb{E}\left[\sum_{i} \frac{\sigma^{4}}{2} C_{S S}^{2}\left(t_{i}, S_{t_{i}}\right) S_{t_{i}}^{4}\left[\frac{\Delta t}{d^{\prime}\left(t_{i}\right)}+O\left(\Delta t^{2}\right)\right]\left(t_{i+1}-t_{i}\right)\right] \\
= & \mathbb{E}\left[\sum_{i} \frac{\sigma^{4}}{2} C_{S S}^{2}\left(t_{i}, S_{t_{i}}\right) S_{t_{i}}^{4} \frac{\Delta t}{d^{\prime}\left(t_{i}\right)}\left(t_{i+1}-t_{i}\right)\right]+\sum_{i} O\left(\Delta t^{3}\right) \\
= & \Delta t \mathbb{E}\left[\sum_{i} \frac{\sigma^{4}}{2} C_{S S}^{2}\left(t_{i}, S_{t_{i}}\right) S_{t_{i}}^{4} \frac{1}{d^{\prime}\left(t_{i}\right)}\left(t_{i+1}-t_{i}\right)\right]+O\left(\Delta t^{2}\right) .
\end{aligned}
$$

We also note that

$$
\mathbb{E}\left[\sum_{i} \frac{\sigma^{4}}{2} C_{S S}^{2}\left(t_{i}, S_{t_{i}}\right) S_{t_{i}}^{4} \frac{1}{d^{\prime}\left(t_{i}\right)}\left(t_{i+1}-t_{i}\right)\right] \rightarrow \mathbb{E}\left[\int_{0}^{T} \frac{\sigma^{4}}{2} C_{S S}^{2}\left(t, S_{t}\right) S_{t}^{4} \frac{1}{d^{\prime}(t)} d t\right]
$$

in $L^{1}$. We claim that the remaining terms from Equation (A.1) will be higher order than $O(\Delta t)$ and thus converge to zero as $\Delta t$ tends to zero. We give the proof for the cross term, and the reasoning for the other terms is similar. Consider the expected value conditioned on $\mathcal{F}_{t_{i}}$ of the cross term

$$
\begin{aligned}
& \mathbb{E}_{\mathcal{F}_{t_{i}}} {\left[\int_{t_{i}}^{t_{i+1}}\left(C_{S}\left(t_{i}, S_{t_{i}}\right)-C_{S}\left(t, S_{t}\right)\right) \mu S_{t} d t \int_{t_{i}}^{t_{i+1}}\left(C_{S}\left(t_{i}, S_{t_{i}}\right)-C_{S}\left(t, S_{t}\right)\right) \sigma S_{t} d W_{t}\right] } \\
& \leq\left(\mathbb{E}_{\mathcal{F}_{t_{i}}}\left[\int_{t_{i}}^{t_{i+1}}\left(C_{S}\left(t_{i}, S_{t_{i}}\right)-C_{S}\left(t, S_{t}\right)\right) \mu S_{t} d t\right]^{2}\right)^{1 / 2} \\
& \times\left(\mathbb{E}_{\mathcal{F}_{t_{i}}}\left[\int_{t_{i}}^{t_{i+1}}\left(C_{S}\left(t_{i}, S_{t_{i}}\right)-C_{S}\left(t, S_{t}\right)\right) \sigma S_{t} d W_{t}\right]^{2}\right)^{1 / 2}
\end{aligned}
$$

using Hölder's inequality. Then by the Itô isometry

$$
\begin{aligned}
&\left(\mathbb{E}_{\mathcal{F}_{t_{i}}}\left[\int_{t_{i}}^{t_{i+1}}\left(C_{S}\left(t_{i}, S_{t_{i}}\right)-C_{S}\left(t, S_{t}\right)\right) \mu S_{t} d t\right]^{2}\right)^{1 / 2} \\
& \qquad\left(\mathbb{E}_{\mathcal{F}_{t_{i}}}\left[\int_{t_{i}}^{t_{i+1}}\left(C_{S}\left(t_{i}, S_{t_{i}}\right)-C_{S}\left(t, S_{t}\right)\right)^{2} \sigma^{2} S_{t}^{2} d t\right]\right)^{1 / 2}\left(\mathbb{E}_{\mathcal{F}_{t_{i}}}\left[\int_{t_{i}}^{t_{i+1}} C_{S S}\left(t_{i}, S_{t_{i}}\right)\left(S_{t}-S_{t_{i}}\right) \mu S_{t} d t+O\left(\Delta t^{2}\right)\right]^{2}\right)^{1 / 2} \\
& \quad \times\left(\mathbb{E}_{\mathcal{F}_{t_{i}}}\left[\int_{t_{i}}^{t_{i+1}} C_{S S}^{2}\left(t_{i}, S_{t_{i}}\right)\left(S_{t}-S_{t_{i}}\right)^{2} \sigma^{2} S_{t}^{2} d t+O\left(\Delta t^{3}\right)\right]\right)^{1 / 2} \\
&=O\left(\Delta t^{3 / 2}\right) O(\Delta t)=O\left(\Delta t^{5 / 2}\right) .
\end{aligned}
$$


Then summing up the terms over all subintervals gives an estimate of

$$
\mathbb{E}\left[\sum_{i} O\left(\Delta t^{5 / 2}\right)\right]=O\left(\Delta t^{3 / 2}\right)>O(\Delta t) .
$$

Lemma A.2. $\quad L_{T} \rightarrow \int_{0}^{T} \sigma^{2} f^{\prime}(0) S_{t}^{3} C_{S S}^{2}\left(t, S_{t}\right) d t$ in $L^{2}$-sense as $\Delta t \rightarrow 0$.

Proof. Define the following sequence of random variables

$$
\Delta_{i} \equiv\left(S_{t_{i}}-S_{t_{i-1}}\right)^{2}-\sigma^{2} S_{t_{i-1}}^{2}\left(t_{i}-t_{i-1}\right) .
$$

After rearranging one gets

$$
\left(S_{t_{i}}-S_{t_{i-1}}\right)^{2}=\Delta_{i}+\sigma^{2} S_{t_{i-1}}^{2}\left(t_{i}-t_{i-1}\right) .
$$

Let us consider

$$
\begin{aligned}
& \sum_{i=1}^{n} C_{S S}^{2}\left(t_{i-1}, S_{t_{i-1}}\right)\left(S_{t_{i}}-S_{t_{i-1}}\right)^{2} S_{t_{i-1}} f^{\prime}(0) \\
= & \sum_{i=1}^{n} C_{S S}^{2}\left(t_{i-1}, S_{t_{i-1}}\right)\left(\Delta_{i}+\sigma^{2} S_{t_{i-1}}^{2}\left(t_{i}-t_{i-1}\right)\right) S_{t_{i-1}} f^{\prime}(0) \\
= & \sum_{i=1}^{n} C_{S S}^{2}\left(t_{i-1}, S_{t_{i-1}}\right) \Delta_{i} S_{t_{i-1}} f^{\prime}(0)+\sum_{i=1}^{n} C_{S S}^{2}\left(t_{i-1}, S_{t_{i-1}}\right) \sigma^{2}\left(t_{i}-t_{i-1}\right) S_{t_{i-1}}^{3} f^{\prime}(0) .
\end{aligned}
$$

It is easy to see that the second sum converges to $\int_{0}^{T} \sigma^{2} f^{\prime}(0) S_{t}^{3} C_{S S}^{2}\left(t, S_{t}\right) d t$. We will show that for the first sum

$$
\sum_{i=1}^{n} C_{S S}^{2}\left(t_{i-1}, S_{t_{i-1}}\right) \Delta_{i} S_{t_{i-1}} f^{\prime}(0) \rightarrow 0 .
$$

We begin by observing that $\mathbb{E}\left[\sum_{i=1}^{n} C_{S S}^{2}\left(t_{i-1}, S_{t_{i-1}}\right) \Delta_{i} S_{t_{i-1}} f^{\prime}(0)\right]^{2}$ consists of diagonal and cross terms. Consider the diagonal terms first:

$$
\begin{aligned}
& \mathbb{E}\left[\sum_{i=1}^{n} C_{S S}^{4}\left(t_{i-1}, S_{t_{i-1}}\right) \Delta_{i}^{2} S_{t_{i-1}}^{2}\left(f^{\prime}(0)\right)^{2}\right] \\
= & \mathbb{E}\left[\sum_{i=1}^{n} C_{S S}^{4}\left(t_{i-1}, S_{t_{i-1}}\right) S_{t_{i-1}}^{2}\left(f^{\prime}(0)\right)^{2} \mathbb{E}_{\mathcal{F}_{t_{i-1}}}\left[\Delta_{i}^{2}\right]\right] .
\end{aligned}
$$

Recall that $S_{t_{i}}=S_{t_{i-1}} e^{Z_{t_{i}}-Z_{t_{i-1}}}$, where $Z_{t}=\bar{\mu} t+\sigma W_{t}$ is a Wiener process with drift $\bar{\mu}\left(=\mu-\frac{1}{2} \sigma^{2}\right)$. Since $Z_{t_{i}}-Z_{t_{i-1}} \sim \mathcal{N}\left(\bar{\mu}\left(t_{i}-t_{i-1}\right), \sigma^{2}\left(t_{i}-t_{i-1}\right)\right)$, then the moment generating function of $Z_{t_{i}}-Z_{t_{i-1}}$ is $M_{\mathcal{N}}(s)=e^{\bar{\mu} s+\sigma^{2} s^{2} / 2}$. Then

$$
\begin{aligned}
& \mathbb{E}_{\mathcal{F}_{t_{i-1}}}\left[\Delta_{i}^{2}\right] \\
= & \mathbb{E}_{\mathcal{F}_{t_{i-1}}}\left[\left(S_{t_{i}}-S_{t_{i-1}}\right)^{2}-\sigma^{2} S_{t_{i-1}}^{2}\left(t_{i}-t_{i-1}\right)\right]^{2} \\
= & \mathbb{E}_{\mathcal{F}_{t_{i-1}}}\left[\left(S_{t_{i}}-S_{t_{i-1}}\right)^{4}-2\left(S_{t_{i}}-S_{t_{i-1}}\right)^{2} \sigma^{2} S_{t_{i-1}}^{2}\left(t_{i}-t_{i-1}\right)+\sigma^{4} S_{t_{i-1}}^{4}\left(t_{i}-t_{i-1}\right)^{2}\right]
\end{aligned}
$$




$$
\begin{aligned}
= & S_{t_{i-1}}^{4}\left[3 \sigma^{4}\left(t_{i}-t_{i-1}\right)^{2}-2\left(1+2 \bar{\mu}\left(t_{i}-t_{i-1}\right)+2 \sigma^{2}\left(t_{i}-t_{i-1}\right)-2\left[1+\bar{\mu}\left(t_{i}-t_{i-1}\right)\right.\right.\right. \\
& \left.\left.\left.\quad+1 / 2 \sigma^{2}\left(t_{i}-t_{i-1}\right)\right]+1\right)\left(t_{i}-t_{i-1}\right) \sigma^{2}+\left(t_{i}-t_{i-1}\right)^{2} \sigma^{4}+O\left(\Delta t^{3}\right)\right] \\
= & 2 S_{t_{i-1}}^{4} \sigma^{4}\left(t_{i}-t_{i-1}\right)^{2}+O\left(\Delta t^{3}\right) .
\end{aligned}
$$

Use this result to rewrite Equation (A.3) as

$$
\begin{aligned}
& \mathbb{E}\left[\sum_{i=1}^{n} C_{S S}^{4}\left(t_{i-1}, S_{t_{i-1}}\right) S_{t_{i-1}}^{2}\left(f^{\prime}(0)\right)^{2} \mathbb{E}_{\mathcal{F}_{t_{i-1}}}\left[\Delta_{i}^{2}\right]\right] \\
& =\mathbb{E}\left[\sum_{i=1}^{n} C_{S S}^{4}\left(t_{i-1}, S_{t_{i-1}}\right) S_{t_{i-1}}^{2}\left(f^{\prime}(0)\right)^{2}\left(2 S_{t_{i-1}}^{4} \sigma^{4}\left(t_{i}-t_{i-1}\right)\left(t_{i}-t_{i-1}\right)+O\left(\Delta t^{3}\right)\right)\right] \\
& =2 \mathbb{E}\left[\sum_{i=1}^{n} C_{S S}^{4}\left(t_{i-1}, S_{t_{i-1}}\right) S_{t_{i-1}}^{6}\left(f^{\prime}(0)\right)^{2} \sigma^{4}\left(\frac{\Delta t}{d^{\prime}\left(t_{i}\right)}+O\left(\Delta t^{2}\right)\right)\left(t_{i}-t_{i-1}\right)\right]+O\left(\Delta t^{2}\right) \\
& =2 \Delta t \mathbb{E}\left[\sum_{i=1}^{n} C_{S S}^{4}\left(t_{i-1}, S_{t_{i-1}}\right) S_{t_{i-1}}^{6}\left(f^{\prime}(0)\right)^{2} \sigma^{4} \frac{1}{d^{\prime}\left(t_{i}\right)}\left(t_{i}-t_{i-1}\right)+\sum_{i=1}^{n} O\left(\Delta t^{2}\right)\right]+O\left(\Delta t^{2}\right) \\
& =2 \Delta t \mathbb{E}\left[\sum_{i=1}^{n} \int_{t_{i-1}}^{t_{i}} C_{S S}^{4}\left(t_{i-1}, S_{t_{i-1}}\right) S_{t_{i-1}}^{6}\left(f^{\prime}(0)\right)^{2} \sigma^{4} \frac{1}{d^{\prime}\left(t_{i}\right)} d t\right]+O\left(\Delta t^{2}\right) \rightarrow 0 \quad(\text { as } \Delta t \rightarrow 0) .
\end{aligned}
$$

This is the only term with the order exactly $O(\Delta t)$. To characterize the convergence we note that

$$
\mathbb{E}\left[\sum_{i=1}^{n} \int_{t_{i-1}}^{t_{i}} C_{S S}^{4}\left(t_{i-1}, S_{t_{i-1}}\right) S_{t_{i-1}}^{6}\left(f^{\prime}(0)\right)^{2} \sigma^{4} \frac{1}{d^{\prime}\left(t_{i}\right)} d t\right] \rightarrow \mathbb{E}\left[\int_{0}^{T} C_{S S}^{4}\left(t, S_{t}\right) S_{t}^{6}\left(f^{\prime}(0)\right)^{2} \sigma^{4} \frac{1}{d^{\prime}(t)} d t\right]
$$

in $L^{1}$. Therefore, we conclude that

$$
\begin{aligned}
& \sum_{i=1}^{n} C_{S S}^{2}\left(t_{i-1}, S_{t_{i-1}}\right)\left(S_{t_{i}}-S_{t_{i-1}}\right)^{2} S_{t_{i-1}} f^{\prime}(0) \\
= & \sum_{i=1}^{n} C_{S S}^{2}\left(t_{i-1}, S_{t_{i-1}}\right) \Delta_{i} S_{t_{i-1}} f^{\prime}(0)+\sum_{i=1}^{n} C_{S S}^{2}\left(t_{i-1}, S_{t_{i-1}}\right) \sigma^{2}\left(t_{i}-t_{i-1}\right) S_{t_{i-1}}^{3} f^{\prime}(0) \\
\rightarrow & \int_{0}^{T} C_{S S}^{2}\left(t, S_{t}\right) S_{t}^{3} \sigma^{2} f^{\prime}(0) d t .
\end{aligned}
$$

Next, we need to show

$$
L_{T}-\sum_{i=1}^{n} C_{S S}^{2}\left(t_{i-1}, S_{t_{i-1}}\right) S_{t_{i-1}} f^{\prime}(0)\left(S_{t_{i}}-S_{t_{i-1}}\right)^{2} \rightarrow 0 .
$$

We omit higher-order term on the way. First we work with

$$
\begin{aligned}
L_{T} & \equiv \sum_{t_{i}<T} \Delta X_{t_{i}}\left[S\left(t_{i}, \Delta X_{t_{i}}\right)-S\left(t_{i}, 0\right)\right] \\
& =\sum_{t_{i}<T} \Delta X_{t_{i}}\left[f\left(\Delta X_{t_{i}}\right)-1\right] S_{t_{i}} \\
& =\sum_{t_{i}<T} \Delta X_{t_{i}}^{2} f^{\prime}(0) S_{t_{i}}+\sum_{t_{i}<T} O\left(\Delta X_{t_{i}}\right)^{3}
\end{aligned}
$$




$$
=\sum_{i=1}^{n} f^{\prime}(0) S_{t_{i}} C_{S S}^{2}\left(t_{i-1}, S_{t_{i-1}}\right)\left(S_{t_{i}}-S_{t_{i-1}}\right)^{2}+\sum_{t_{i}<T} O\left(\Delta X_{t_{i}}\right)^{3} .
$$

Now we turn our attention to

$$
\begin{aligned}
& \mathbb{E}\left[L_{T}-\sum_{i=1}^{n} f^{\prime}(0) S_{t_{i-1}} C_{S S}^{2}\left(t_{i-1}, S_{t_{i-1}}\right)\left(S_{t_{i}}-S_{t_{i-1}}\right)^{2}\right]^{2} \\
& =\mathbb{E}\left[\sum_{i=1}^{n} f^{\prime}(0) C_{S S}^{2}\left(t_{i-1}, S_{t_{i-1}}\right)\left(S_{t_{i}}-S_{t_{i-1}}\right)^{3}+\sum_{t_{i}<T} O\left(\Delta X_{t_{i}}\right)^{3}\right]^{2}=O\left(\Delta t^{2}\right) \rightarrow 0 .
\end{aligned}
$$

Therefore,

$$
\begin{aligned}
L_{T} & =L_{T}-\sum_{i=1}^{n} f^{\prime}(0) S_{t_{i-1}} C_{S S}^{2}\left(t_{i-1}, S_{t_{i-1}}\right)\left(S_{t_{i}}-S_{t_{i-1}}\right)^{2} \\
& +\sum_{i=1}^{n} f^{\prime}(0) S_{t_{i-1}} C_{S S}^{2}\left(t_{i-1}, S_{t_{i-1}}\right)\left(S_{t_{i}}-S_{t_{i-1}}\right)^{2} \\
& \rightarrow \int_{0}^{T} \sigma^{2} f^{\prime}(0) S_{t}^{3} C_{S S}^{2}\left(t, S_{t}\right) d t
\end{aligned}
$$

The proof of the lemma is finished.

By combining the lemmas, we now prove Theorem 2.1.

Proof. Rearranging the terms of the PDE one gets

$$
\begin{gathered}
\sigma^{2} f^{\prime}(0) S^{3} C_{S S}^{2}=-C_{t}-1 / 2 \sigma^{2} S^{2} C_{S S} \\
-\int_{0}^{T} \sigma^{2} f^{\prime}(0) S^{3} C_{S S}^{2} d t=\int_{0}^{T}\left[-C_{t}-1 / 2 \sigma^{2} S^{2} C_{S S}\right] d t .
\end{gathered}
$$

Then, lemmas A.1 and A.2 imply

$$
\begin{aligned}
& C\left(0, S_{0}\right)+\sum_{t_{i}<T} C_{S}\left(t_{i}, S_{t_{i}}\right)\left(S_{t_{i+1}}-S_{t_{i}}\right)-\sum_{t_{i}<T} \Delta X_{t_{i}}\left[S\left(t_{i}, \Delta X_{t_{i}}\right)-S\left(t_{i}, 0\right)\right] \\
\longrightarrow & C\left(0, S_{0}\right)+\int_{0}^{T} C_{S}\left(t, S_{t}\right) d S_{t}-\int_{0}^{T} \sigma^{2} f^{\prime}(0) S_{t}^{3} C_{S S}^{2}\left(t, S_{t}\right) d t \\
= & C\left(0, S_{0}\right)+\int_{0}^{T} C_{S}\left(t, S_{t}\right) d S_{t}+\int_{0}^{T}\left[C_{t}+1 / 2 \sigma^{2} S^{2} C_{S S}\right] d t \\
= & C\left(T, S_{T}\right)=p\left(S_{T}\right)
\end{aligned}
$$

by Itô's formula, which completes the proof of Theorem 2.1.

Finally, we are ready to prove Theorem 2.2.

Proof. Note that we omit the higher order terms. Denote

$$
\begin{aligned}
& \sum_{1} \equiv \sum_{t_{i}<T} C_{S}\left(t_{i}, S_{t_{i}}\right)\left(S_{t_{i+1}}-S_{t_{i}}\right) \\
& \sum_{2} \equiv L_{T}=\sum_{t_{i}<T} \Delta X_{t_{i}}\left(S\left(t_{i}, \Delta X_{t_{i}}\right)-S\left(t_{i}, 0\right)\right)
\end{aligned}
$$




$$
\begin{aligned}
& \int_{1} \equiv \int_{0}^{T} C_{S}\left(t, S_{t}\right) d S_{t} \\
& \int_{2} \equiv \int_{0}^{T} \sigma^{2} f^{\prime}(0) S_{t}^{3} C_{S S}^{2}\left(t, S_{t}\right) d t .
\end{aligned}
$$

With this notation, the mean squared hedging error is written as

$$
\begin{aligned}
& \mathbb{E}\left[C\left(0, S_{0}\right)+\sum_{t_{i}<T} C_{S}\left(t_{i}, S_{t_{i}}\right)\left(S_{t_{i+1}}-S_{t_{i}}\right)-\sum_{t_{i}<T} \Delta X_{t_{i}}\left(S\left(t_{i}, \Delta X_{t_{i}}\right)-S\left(t_{i}, 0\right)\right)-p\left(S_{T}\right)\right]^{2} \\
= & \mathbb{E}\left[\left(\sum_{1}-\sum_{2}-\left(\int_{1}-\int_{2}\right)\right)^{2}\right] \\
= & \mathbb{E}\left[\left(\sum_{1}-\int_{1}\right)^{2}-2\left(\sum_{1}-\int_{1}\right)\left(\sum_{2}-\int_{2}\right)+\left(\sum_{2}-\int_{2}\right)^{2}\right] .
\end{aligned}
$$

The estimates for the first and third terms in the expectation are obtained from lemmas A.1 and A.2. Only the term in the middle of the expression needs to be investigated. By omitting the higher order terms and the almost identical computations to the proofs of the lemmas, the cross term of the errors is essentially

$$
\begin{aligned}
& \mathbb{E}\left[\sum_{i} \int_{t_{i-1}}^{t_{i}}\left(C_{S}\left(t, S_{t}\right)-C_{S}\left(t_{i-1}, S_{t_{i-1}}\right)\right) d S_{t} C_{S S}^{2}\left(t_{i-1}, S_{t_{i-1}}\right) \Delta_{i} S_{t_{i-1}} f^{\prime}(0)\right] \\
= & \mathbb{E}\left[\sum_{i} C_{S S}^{3}\left(t_{i-1}, S_{t_{i-1}}\right) S_{t_{i-1}} f^{\prime}(0) \mathbb{E}_{\mathcal{F}_{t_{i-1}}}\left[\Delta_{i} \int_{t_{i-1}}^{t_{i}}\left(S_{t}-S_{t_{i-1}}\right) d S_{t}\right]\right] \\
=\mathbb{E} & {\left[\sum_{i} C_{S S}^{3}\left(t_{i-1}, S_{t_{i-1}}\right) S_{t_{i-1}}^{5} f^{\prime}(0) \sigma^{4}\left(t_{i}-t_{i-1}\right)^{2}\right] } \\
=\mathbb{E} & {\left[\sum_{i} C_{S S}^{3}\left(t_{i-1}, S_{t_{i-1}}\right) S_{t_{i-1}}^{5} f^{\prime}(0) \sigma^{4}\left(\frac{\Delta t}{d^{\prime}\left(t_{i-1}\right)}+O\left(\Delta t^{2}\right)\right)\left(t_{i}-t_{i-1}\right)\right] } \\
& \longrightarrow \Delta t \mathbb{E}\left[\int_{0}^{T} C_{S S}^{3}\left(t, S_{t}\right) S_{t}^{5} f^{\prime}(0) \sigma^{4} \frac{1}{d^{\prime}(t)} d t\right] .
\end{aligned}
$$

The result follows by collecting the estimates obtained.

Acknowledgement. The authors are grateful for the editor's recommendation and helpful comments.

\section{REFERENCES}

[1] R. Almgren, Optimal execution with non-linear impact functions and trading-enhanced risk, Applied Mathematical Finance, 10, 1-18, 2003.

[2] R. Almgren and N. Chriss, Optimal execution of portfolio transactions, J. of Risk, 3, 5-39, 2001.

[3] M Avellaneda and M.D. Lipkin, A market-induced mechanism for stock pinning, Quantitative Finance, 3, 417-425, 2003.

[4] P. Bank and D. Baum Hedging and portfolio optimization in financial markets with a large trader, Mathematical Finance, 14, 1-18, 2004. 


\section{OPTION REPLICATION IN DISCRETE TIME WITH THE COST OF ILLIQUIDITY}

[5] U. Cetin, R.A. Jarrow, and P. Protter, Liquidity risk and arbitrage pricing theory, Finance and Stochastics, 8, 311-341, 2004.

[6] U. Cetin, H.M. Soner, and N. Touzi, Option hedging for small investors under liquidity costs, Finance and Stochastics, 14, 317-341, 2010.

[7] P.A. Forsyth, A Hamilton-Jacobi-Bellman approach to optimal trade execution, Appl. Numerical Math., 61, 241-265, 2011.

[8] R. Frey, Perfect option hedging for a large trader, Finance and Stochastics, 2, 115-141, 1988.

[9] R. Frey and A. Stremme, Market volatilities and feedback effects from dynamic hedging, Mathematical Finance, 7, 351-374, 1997.

[10] E.R. Grannan and G.H. Swindle, Minimizing transaction costs of option hedging strategies, Mathematical Finance, 6, 341-364, 1996.

[11] R.A. Jarrow, Derivative security markets, market manipulation and option pricing theory, Journal of Financial and Quantitative Analysis, 241-261, 1994.

[12] H.Ku, K. Lee, and H. Zhu, Discrete time hedging with liquidity risk, Finance Research Letters, 9 , 135-143, 2012.

[13] H.E. Leland, Option pricing and replication with transaction costs, The Journal of Finance, 40, 1283-1301, 1985.

[14] F. Longstaff, Optimal portfolio choice and the valuation of illiquid securities, Review of Financial Studies, 14, 403-431, 2001.

[15] E. Platen and M. Schweizer, On feedback effects from hedging derivatives, Mathematical Finance, 8, 67-84, 1998.

[16] L.C.G. Rogers and S. Singh, The cost of illiquidity and its effects on hedging, Mathematical Finance, 20, 597-615, 2010.

[17] S.E. Shreve, Stochastic Calculus for Finance: Continuous-Time Models, Springer-Verlag, 2004. 